\section{Complexity still running}

SIR - Your correspondents (Nature 348, $280 ; 1990)$ trying to tackle my question about runaway complexity (Nature 347, 704 ; 1990) have missed the point, which concerns the inverse relationship between morphological complexity and population size. Invoking the eukaryote/prokaryote distinction is not 'the simple answer' as offered by Philip Wood, because the origin of eukaryotes from prokaryotes is itself only part of the more general problem. I accept, of course, the claim of great biochemical versatility, adaptability and general success of today's prokaryotes, made on their behalf by an unusually articulate variant of $E$. coli (Nature 349, 97; 1991).

One might loosely define more complex organisms as having more morphological features whose presence is ascribed to the sometime action of directional selection. But more complex organisms generally have smaller population sizes and tend to have longer generation times, so en route to increasing complexity there should be fewer and fewer opportunities to build up any new complex feature step-by-step in a sustained series of selection events. Most scenarios to generate the phenomenal complexity of even the simplest metazoans seem to require vast numbers of successive selection events acting on particular traits; that is, episodes of persistent directional selection for one feature after another. The awesome morphological complexity of organisms such as vertebrates that have far fewer individuals on which selection can act therefore remains somewhat puzzling (for me at least), despite the geological timescales available and notwithstanding the insights provided by John Bonner in his book The Evolution of Complexity and Richard Dawkins' demonstration of the power of selection in The Blind Watchmaker. Could there therefore be a tendency, as yet neither fully recognized nor understood, for more complex organisms to throw up ever wider ranges of viable variation, including occasional novel but well-integrated features that give every impression of sustained selection? If so, any such tendency for 'runaway complexity' may have had a relatively free rein of expression during the metazoan radiations in the late Precambrian and early Cambrian.

In general, we know much about frequency-changing processes in evolution but still very little about how new traits and forms arise, as recently admitted by Endler and McLellan in a comprehensive review ( $A$. Rev. Ecol. Syst. 19, 395-421; 1988).

Peter R. Sheldon

Department of Geology,

University of Wales,

PO Box 914 .

Cardiff CF1 $3 Y E$, UK
Origins of AIDS

SIR - Why do Western analysts need always to invoke exotic sexual practices by Africans in order to explain transmission of HIVrelated viruses from monkeys to man when far likelier routes for transmission are readily apparent? In an otherwise excellent letter on the origin and spread of AIDS, A. Karpas (Nature $348578 ; 1990$ ) cites a paper by F. Noireau (Lancet i, 1498; 1987) suggesting that elaborate sexual rituals involving human genitalia and monkey blood are a likely mode of trans-species transmission. Yet in the same letter, Karpas discusses monkey-virus transmission to (Western) laboratory workers. Suggestions of deviant sexual behaviour are no longer evoked simple monkey bites are now considered sufficient to explain transmission. Man has been hunting, killing and eating monkey in Africa for millennia. This has undoubtedly led to countless bites, cuts and abrasions that have provided ideal opportunities for bloodborne inoculation. Scientific writers should leave descriptions of the more exotic sexual rituals to either anthropologists or the glossier pages of other types of publication, rather than Nature.

Department of Microbiology,

JOHNJOE MCFADDEN

University of Surrey,

Guildford, Surrey GU2 5XH, UK

\section{Genome project}

SIR - Biologists should be disquieted by the special pleading of Walter Gilbert for the genome project (Nature 349, 99; 1991). The assumptions he makes are odd. For instance, he seems to imply that, once we have identified the genes in an organism, we have obtained virtually all the basic information required to explain biological phenomena. The limiting factor is understanding not only the gene structure but also the gene expression, which varies in a most complex way. Monod made great advances towards the understanding of gene expression but only to one case, namely expression of the $\beta$-galactosidase gene.

But consider the problem when one becomes seriously ill with, say, a virus infection. Although the genes are the same, a cascade or even catastrophic series of changes occurs in the body functions, or gene expressions, of which man is essentially ignorant. The 'new paradigm' should be addressed not merely to gene structure but to elucidation of the integrated functions of genes, enzymes, membranes and metabolites in the whole organism. Only such an extended paradigm can lead to explanations of the biological phenomena that Gilbert lists in his preamble. Incidentally, formulating a hypothesis and testing it experimentally is not due to start only when we have identified the genes. A review of the literature will show that biologists have always effectively used that principle.
The use of 'kits' and computers on the grand scale for the human genome project is an admirable ambition. But let us recognize its limitations and ensure that it does not distort the rest of bioscientific research. The attack on the gene expression problem is much more challenging and difficult. The resort to the kit and the computer routines seems to be an example of that well-known human failing - taking the line of least resistance.

S. JOHN PIRT

(Emeritus professor, King's College London)

50 Chartfield Avenue, London SW15 6HG. UK

\section{Grants as status}

SiR - Ray lles (Nature 349, 188; 1991) is lucky to be able to court the biotechnology companies. Obvious sources of funding in anaesthesia are the pharmaceutical companies - except that their money does not count in the scheme of 'brownie points' errected by those who judge our worth, to whom the acquisition of grant money seems the most important criterion. However, in these days of shrinking resources one would imagine that doing research for the least cost would be a laudable aim; yet one scores more highly by securing grant money, even if the project can be done without its need. It is an intellectually bankrupt notion to suppose that research that is more expensive is necessarily better. I pity the theoreticians; or perhaps they secure grants for supplies of expensive fountain pens and pure vellum on which to write.

Neville W. Goodman University Department of Anaesthesia, Southmead Hospital, Bristol, BS10 5NB, UK

\section{Ordered layers}

SIR - The intriguing piece by Daedalus on ambitious chemists and micromagnetics (Nature 348, 588; 1990) came in the middle of a related discussion here. I have supposed that, if the vapour phase deposition of organic compounds (such as porphyrins, phthalocyzanines and other aromatics) which are able to sustain a sizeable induced ring current were to be carried out in a high magnetic field, then the induced magnetic field would influence the mode of deposition. With care it might be possible to get nicely ordered layers in this way; and clearly the experiment could be done with the external field at various orientations to the direction of gaseous molecular flow.

Has this sort of experiment been done? If not, would anyone be prepared to do it? If not in the United Kingdom, then maybe in Japan? I would be happy to supply suitable porphyrins and phthalocyanines.

R.BONNETT

Department of Chemistry,

Queen Mary and Westfield College,

Mile End Road, London E1 4NS, UK 